\title{
VIEWPOINT
}

\section{Thirty years of critical care medicine}

\author{
Jean-Louis Vincent ${ }^{* 1}$, Mervyn Singer ${ }^{2}$, John J Marini ${ }^{3}$, Rui Moreno ${ }^{4}$, Mitchell Levy ${ }^{5}$, Michael A Matthay ${ }^{6}$, Michael Pinsky, \\ Andrew Rhodes ${ }^{8}$, Niall D Ferguson ${ }^{9}$, Timothy Evans ${ }^{10}$, Djillali Annane ${ }^{11}$ and Jesse B Hall12
}

\begin{abstract}
Critical care medicine is a relatively young but rapidly evolving specialty. On the occasion of the 30th

International Symposium on Intensive Care and

Emergency Medicine, we put together some thoughts

from a few of the leaders in critical care who have been actively involved in this field over the years. Looking back over the last 30 years, we reflect on areas in which, despite large amounts of research and technological and scientific advances, no major therapeutic breakthroughs have been made. We then look at the process of care and realize that, here, huge progress has been made. Lastly, we suggest how critical care medicine will continue to evolve for the better over the next 30 years.
\end{abstract}

Every day you may make progress. Every step may be fruitful. Yet there will stretch out before you an ever-lengthening, ever-ascending, ever-improving path. You know you will never get to the end of the journey. But this, so far from discouraging, only adds to the joy and glory of the climb.

Winston Churchill

\section{Introduction}

Critical care medicine is a relatively young discipline that has rapidly grown into a full-fledged specialty. Demand for intensive care has steadily escalated, and the ratio of intensive care unit (ICU) to hospital beds is increasing everywhere. ICUs now hold a key position in all hospitals, and critical care physicians are responsible for managing the ever-increasing numbers of patients with complex, life-threatening medical and surgical disease. Perhaps nowhere else in clinical medicine has the evolution of technology and scientific advance been so apparent and

*Correspondence: jlvincen@ulb.ac.be

'Department of Intensive Care, Erasme Hospital, Université libre de Bruxelles,

Route de Lennik 808, 1070 Bruxelles, Belgium

Full list of author information is available at the end of the article new ideas, concepts, and discoveries moved so fast from bench to bedside. On the occasion of the 30th International Symposium on Intensive Care and Emergency Medicine, we thought it would be instructive to put together some thoughts from a few of the leaders in critical care who have been actively involved in this field over the years. However, as with many anniversaries, we look back over the last 30 years with mixed feelings. Despite considerable technological and scientific advances, we cannot help but feel a little disappointed that our discipline has made few ground-shaking steps forward, especially in therapeutics. Nevertheless, we should be pleased with the progress and improvements that have been made, notably in the process of care.

We have not made much progress in therapeutics ... To be honest, there have been very few major developments in critical care in terms of specific new treatments and cures over the last 30 years. Our success in translating the many advances in basic scientific knowledge and understanding of the pathobiology of syndromes, such as sepsis and acute respiratory distress syndrome (ARDS), to pharmacologic or biologic therapies in order to interrupt injurious processes has been minimal, and this is due in part to the complex and variable nature of these disease processes, the heterogeneous nature of the patients who are affected, and the inadequate preclinical models currently available [1]. No 'magic bullets' that have directly saved lives in heterogeneous groups of patients have been developed. Many prospective multicenter randomized trials have been conducted; in itself, this may be viewed as progress and evidence of increasing maturity. However, the vast majority of these trials have failed to demonstrate improved outcomes with the intervention under investigation [2]. Even the encouraging findings of single-center studies have not been reproduced in later multicenter trials: a good example of this is the concept of tight blood sugar control, in which the results from the initial singlecenter study [3] could not be reproduced by the multicenter VISEP (Volume Substitution and Insulin Therapy in Severe Sepsis) [4], Glucontrol [5], or NICESUGAR (Normoglycemia in Intensive Care Evaluation and Survival Using Glucose Algorithm Regulation) [6] 
studies. There are many reasons for the apparent failure of randomized controlled trials to demonstrate improved outcomes with the interventions that have been tested: for example, the interventions were simply not effective, the studies were underpowered, and the selected mortality endpoint is inadequate or inappropriate. However, the main reason is likely related to the logistics of multicenter trials, which require the inclusion of a broad spectrum of patients and loose co-intervention controls.

If we consider just a few of the main areas of critical care medicine, the (limited) progress made in the last 30 years seems disappointingly obvious:

- Sepsis: Perhaps our main advance in the field of sepsis has been the unraveling and greater understanding of the pathogenetic response, which offered hope for the development of effective therapies for sepsis. Unfortunately, only activated protein $\mathrm{C}(\mathrm{aPC})$ has actually been licensed for use in such patients, and the efficacy of this drug has been challenged. Numerous other antisepsis therapies have been tested, many in large multicenter phase III studies, yet have failed to show overall effectiveness in improving patient outcomes.

Much has been said about the importance of early diagnosis of sepsis and the potential role of biomarkers, but we remain frustrated in our attempts to identify biomarkers that are specific for sepsis and that can be used for diagnosis, therapeutic guidance, or prognostication. The role of immunomodulatory nutritional solutions has also not been clarified. Whether specialized nutrients, such as glutamine or omega-3 fatty acids, are beneficial remains uncertain. Apart from the effects of selenium on the reduction of secondary bacterial infection, no consistent effect has been shown for other drugs, such as glutamine (Peter Andrews, SIGNET [Scottish Intensive Care Glutamine or Selenium Evaluative Trial], personal communication).

- Respiratory failure and ARDS: Progress has been made in the use of noninvasive mechanical ventilation, which is now widely employed and for which indications have been more clearly defined. Arguably, we have made major progress in the ventilatory treatment of patients with ARDS over the past 30 years through the recognition and avoidance of iatrogenic ventilatorinduced lung injury (VILI) by limiting tidal volumes and airway pressures [7]. However, we still have much to learn about the optimal ventilatory management of patients with ARDS. Less aggressive ventilation has clearly resulted in a reduced incidence of barotrauma, yet debate persists over the best lung protective ventilation strategy and how to optimally apply positive end-expiratory pressure (PEEP). We now have some evidence, albeit not strong, that fluid balance is an important determinant of outcome in patients with acute lung injury (ALI), although our ability to accurately define a level of preload to which fluid therapy should be titrated remains elusive. Turning patients to the prone position also appears to be associated with reduced mortality rates in the most severe cases. Disappointingly, no specific pharmacologic intervention showing clear outcome benefit has been forthcoming, with approaches ranging from inhaled surfactant or nitric oxide to systemic administration of antioxidants or anti-inflammatory agents. Although most studies do not show a clear benefit of steroids in ARDS, their precise role remains controversial in these patients. Even though mortality rates may be decreasing [8], we are still left with many unanswered questions.

- Cardiovascular diseases: There has been considerable progress in the management of acute myocardial infarction with early thrombolysis and percutaneous coronary intervention, although these are often applied outside the ICU. Although minor modifications are endorsed on an almost yearly basis, cardiopulmonary resuscitation has not been shown to increase the number of lives saved, especially in patients already in the hospital. The development of ultrasound for cardiovascular diagnosis and monitoring has been a major advance, but we have made less progress regarding hemodynamic support of the failing circulation. We still rely on the same catecholamines, such as epinephrine, norepinephrine, and dobutamine. The use of dopamine for renal support and as a firstline vasopressor agent has waned, but it has not been convincingly replaced by other drugs. The problem of 'vasoparesis' (resistance vessels unresponsive to catecholamines) is unresolved. We have rediscovered vasopressin, but there is much debate about its potentially beneficial effects. The introduction of phosphodiesterase inhibitors or levosimendan has not yielded major outcome benefits. We still await reliable agents that selectively improve ventricular function without risking ischemia, tachycardia, or unwanted vasoactive and other effects. Selective and titratable agents to control heart rate which do not adversely affect ventricular performance are also lacking, and how to improve right ventricular dysfunction and address pulmonary hypertension remain major unsolved problems.

- Renal system: We now have a far greater understanding than before of the causes of acute kidney injury (AKI); however, this has not resulted in the development of effective renal protective strategies. Hemodialysis or hemofiltration or both in various modalities are now routinely offered to critically ill patients with acute renal failure, yet randomized multicenter trials have not clearly established that one form of renal support or level of intensity over another impacts on patient 
outcomes [9]. Although the development of continuous veno-venous hemofiltration $(\mathrm{CVVH})$ with or without associated dialysis could be seen as an advance because it greatly facilitates fluid management and the provision of adequate nutrition, it has not been shown to be clearly superior to intermittent dialysis in terms of outcome.

- Coagulation/anticoagulation: While low-molecularweight heparins offer some functional advantages over unfractionated heparin and recently introduced alternatives, such as argatroban and leparudin, help obviate the risk of heparin-induced consequences, none has usurped the primacy of the heparins in delivering therapeutic anticoagulation within the ICU. Catheter-based interventions, such as locally infused thrombolytics and mechanical ablation, now help when anticoagulants alone are insufficient or contraindicated in the treatment of life-threatening thromboembolism. The development of recombinant factor VIIa was initially hailed as a breakthrough to help limit bleeding; however, studies have shown only a reduction in the use of transfusions and that benefit may be negated by an increased risk of thromboembolic events. Hence, the European Medicines Agency (EMEA) has issued a specific warning that the drug should not be used outside its approved indications.

- Neurological system: Advances have been made in terms of neuro-monitoring modalities and in treatments for specific neurological disease (for example, thrombolytic therapy for ischemic stroke and therapeutic cooling after cardiac arrest). However, there have been relatively few advances in the approach to many other neurological processes requiring intensive care (for example, traumatic brain injury), and mortality and morbidity rates in such patients remain high. The development of new drugs for neurological disorders has been particularly disappointing.

\section{Too many syndromes?}

By describing new entities and coining new syndromes, we thought that diagnosis would be more specific and studies could be performed more easily on more homogeneous groups of patients, thus aiding and abetting the development of new therapies. However, this may not be the case. For example, introducing the concept of the systemic inflammatory response syndrome (SIRS) did not prove to be helpful, and whether the AKI approach is really better than acute renal dysfunction or failure is not at all certain. It could even be argued that existing definitions of ALI and ARDS have not resulted in better management given that the only positive study outcome is that we should limit tidal volumes and plateau airway pressure in patients meeting these criteria. We have ended up grouping many heterogeneous patients together; this may have contributed to our lack of therapeutic progress in this area.

\section{Is less better?}

Undoubtedly, we have learned over the past 30 years that more is not necessarily better. We have, in fact, realized that fewer interventions or less of a particular intervention is frequently associated with better outcomes. Previously, a primary goal of acute care management was to restore all measured variables to their 'normal' values whether they were laboratory values, such as electrolytes, blood gases, or hematocrit, or physiological values, such as cardiac output or urine output. For example, we now use fewer blood transfusions since the multicenter Canadian study by Hebert and colleagues [10] that noted that a hemoglobin transfusion trigger of $7 \mathrm{~g} / \mathrm{dL}$ resulted in no increase in mortality when compared with transfusions to a hemoglobin of greater than $9 \mathrm{~g} / \mathrm{dL}$. Invasive hemodynamic monitoring (for example, the pulmonary artery catheter) has been largely replaced by technologies that are less invasive, even though these lack direct measures of pulmonary vascular pressures and mixed venous oxygen saturation.

Thanks to the development of interventional radiology, numerous therapeutic interventions that once required surgery are now accomplished less invasively. Abscess drainage, stent placement, interruption of torrential gastrointestinal bleeding, coiling of intracerebral aneurysms, and percutaneous coronary intervention are only a few salient examples. Mechanical ablation or localized infusion of thrombolytics can safely accomplish clot lysis in the setting of massive pulmonary embolism, often taking the place of surgical embolectomy or systemic thrombolysis. Loculated pleural effusions and empyemas that once required thoracotomy for drainage can often be addressed by localized instillation of a fibrinolytic through a well-placed drainage catheter. When such problems cannot be addressed in this way, video-assisted thoracoscopic (rather than open thorax) procedures are quite often successful.

Lower tidal volumes are widely used in mechanically ventilated patients [7], and invasive mechanical ventilation is increasingly replaced by noninvasive ventilatory techniques, especially in acute-on-chronic respiratory failure and for immunosuppressed patients; nonetheless, its role in the treatment of patients with acute respiratory failure outside experimental settings continues to be controversial. Sedation is used less routinely and in lower doses; we now recognize that, whereas in the past most patients on mechanical ventilation were heavily sedated, using less sedation can facilitate weaning, prevent delirium and post-traumatic stress disorder, and reduce lengths of ICU stay and associated costs [11]. The story of 


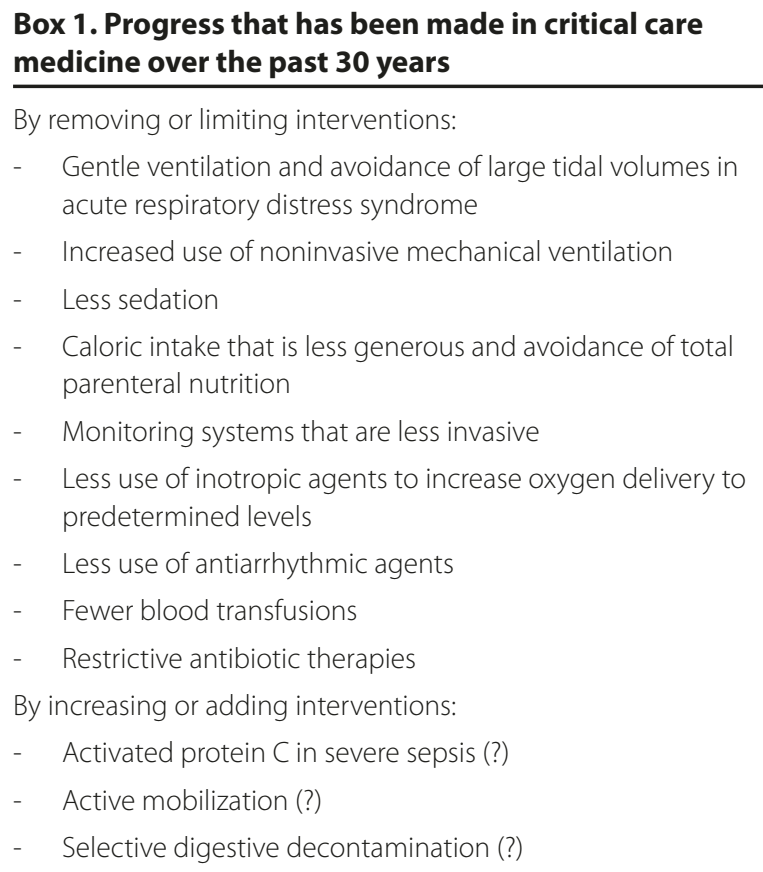

By removing or limiting interventions:

Gentle ventilation and avoidance of large tidal volumes in acute respiratory distress syndrome

Increased use of noninvasive mechanical ventilation

Less sedation

Caloric intake that is less generous and avoidance of total parenteral nutrition

Monitoring systems that are less invasive

Less use of inotropic agents to increase oxygen delivery to predetermined levels

Less use of antiarrhythmic agents

Fewer blood transfusions

Restrictive antibiotic therapies

By increasing or adding interventions:

Activated protein C in severe sepsis (?)

Active mobilization (?)

Selective digestive decontamination (?)

weaning strategies has followed a similar trajectory. Many studies evaluated complex variables and optimal methods of orchestrating the transition to spontaneous breathing. However, facilitated weaning has become the preferred, minimalist approach; when simple criteria are met (reversal or improvement in the reason for initiation of ventilation, absence of severe hypoxemia, relative hemodynamic stability, and an adequate level of consciousness), the ventilator is simply stopped and the patient is placed on a T-piece or minimal pressure support for 30 minutes and then reassessed; things could not be more elementary.

We have learned (perhaps the hard way, through our mistakes) how inappropriate or excessive use of potent antibiotics may lead to increased prevalence of antimicrobial resistance. Many ICUs are now faced with multiple organisms that are resistant to many of our common antibiotics. We have also learned that specific infections, such as ventilator-associated pneumonia (VAP), can be cured by a shorter course of antibiotics.

Feeding has also become simpler, with fewer calories and fewer specialized nutrients. Fewer chest radiographs are performed, arterial blood gas measurements are less frequently requested, and the ventilator circuitry is changed less frequently. We now tolerate greater degrees of physiological abnormality in the critically ill (for example, in carbon dioxide, hemoglobin, and blood pressure) rather than drive the patient harder to achieve 'normal' values. Clearly, multiple aspects of intensive care management have become less invasive and less intensive (Box 1).

\section{... but we have made considerable progress in other aspects of patient management}

Although no huge leaps have been made in new therapies for intensive care patients, marked advances have been made in the process of care. These advances, when implemented, can impact less directly, but no less importantly, on patient outcomes.

- Critical care medicine has established itself as a specialty in its own right, and the importance of intensivist-led care in optimizing outcomes has been demonstrated. The approach to patient care has gradually evolved from a rather paternalistic, physician-directed process to a comprehensive, multidisciplinary, multiprofessional team approach. Regular bedside rounds and 24-hour intensivist-led care have been associated with better outcomes. Unquestionably, the formation of multidisciplinary teams has improved care delivery. Nurses, physiotherapists, pharmacists, and other team members are increasingly responsible for executing management protocols, including weaning, sedation, nutrition, glucose control, vasopressor and electrolyte manipulation, patient positioning, and early ambulation. Checklists such as the FASTHUG (Feeding, Analgesia, Sedation, Thromboembolic prophylaxis, Head-of-bed elevation, stress Ulcer prevention, and Glucose control) [12] have been introduced to encourage this team approach and to provide a simple mnemonicbased reminder of the important 'routine' aspects of patient care. Goal-directed orders are increasingly common. Protocolized care has been advocated, although not all agree that it is beneficial and it remains a subject of intense debate. The same is true for the use of guidelines to standardize care [13].

- We recognize that 'time is tissue' and that early effective management is crucial to maximize patient outcomes in all disease processes, including trauma management, percutaneous coronary intervention for myocardial infarction, early administration of adequate fluids and appropriate antibiotics in sepsis, early thrombolysis in stroke, and perioperative hemodynamic optimization.

- We now manage fluid balance more effectively, administering more fluids in the acute resuscitation phase and then more actively removing excess fluids later on, when the patient has stabilized. The development of CVVH has helped in this regard. A conservative fluid strategy adopted once the patient is no longer in shock results in faster weaning from mechanical ventilation in ARDS patients [14].

- The approach to patient care is more humane and personal. The ICU is much more open to visits by family members. Communication with patients and relatives has certainly improved. Ethical issues, 
including decisions on end-of-life care, are also more openly discussed.

- We mobilize our patients better and sooner, leading to reduced risks of VAP, thrombophlebitis, decubitus ulcers, and delirium.

- We have identified critical care as one important piece in a complex continuum of care and recognize its holistic nature. Few other specialties deal with the whole body, including the mind. The physiological and psychological aspects of critical illness, the recovery processes (both short- and long-term), and the impact upon not only the patient but their loved ones are increasingly appreciated and managed.

- We pay more attention to avoiding potential errors, to encouraging error reporting, and to managing errors better when they occur, having learned from the airline industry how to deal with these complex and occasionally fraught situations (crew resource management). Increased use of electronic medical records and prescriptions may also help reduce errors.

- We have begun to evaluate the limited evidence available to support some established therapies and question their place in modern intensive care. Studies have been conducted to evaluate issues of ongoing uncertainty, such as the safety of albumin [15], the pulmonary artery catheter [16], and dopamine as a first-line agent in shock [17], providing important information on some of the many aspects of clinical practice which are widely used but unproven.

- We are more aware of the risks of nosocomial infection and the importance of preventive measures (starting with good hygiene, including hand washing), which we are applying more routinely and more effectively.

- We understand better the determinants of mortality in the patient with critical illness, in particular the roles of prior diseases and of the presence, degree, pattern, and evolution of multiple organ dysfunction/failure. We have achieved a better understanding of underlying disease processes, including the complex pathophysiology of sepsis, the heterogeneous nature of ARDS, the important role of the intra-abdominal compartment syndrome, and more subtle matters such as increased awareness of relative adrenal or vasopressin insufficiency or both in patients in circulatory shock.

- We have learned much about the epidemiology of critical illness. We have complemented single-center, physiologically focused, and mechanism-probing investigations with national and international collaborative studies centered on effectiveness. Large multicenter and multinational registries have appeared and evolved for purposes of benchmarking and quality assurance (for example, ICNARC [Intensive Care National Audit and Research Centre], GiViTi [Gruppo Italiano per la Valutazione degli interventi in Terapia
Intensiva], and ASDI [Austrian Center for Documentation and Quality Assurance in Intensive Care Medicine]) or for purposes of research (for example, ANZICS [Australian and New Zealand Intensive Care Society] or ESICM [European Society of Intensive Care Medicine] flu registries). Several large national and international consortiums (for example, ARDSNet, Canadian Critical Care Trials Group [CCCTG], ANZICS, Sepsis Occurence in Acutely ill Patients [SOAP], and European Critical Care Research Network [ECCRN]) have been created to facilitate the performance of large multicenter clinical trials and observational studies to address important questions.

- International collaboration between experts and scientific societies in programs such as the Surviving Sepsis Campaign has highlighted the importance of critical illness and led to the development of evidencebased guidelines for sepsis [18] and, importantly, mechanisms to assess the effectiveness of their implementation.

\section{The pendulum of medicine}

As we look back over the past 30 years, we frequently see evidence of the so-called pendulum effect. Clinical trials of several interventions have yielded apparently conflicting, even opposing, results as the pendulum has swung from a benefit effect through no effect to potential harm and then all the way back to benefit, leaving the practicing clinician rather confused. We can offer several examples:

- Forty years ago, high-dose steroids were administered in sepsis for their anti-inflammatory properties [19]. Studies then suggested that, in fact, steroids were ineffective or even potentially harmful and so their use in sepsis decreased. Subsequent trials then suggested that smaller doses could help reduce vasopressor requirements in patients with septic shock and possibly reduce mortality. However, a large international multicenter study failed to confirm these results [20], and steroid use in sepsis has again decreased. We are currently left with a recommendation to consider the use of steroids in only the most severe forms of septic shock despite strong discussion about the risk/benefit cutoff [18].

- Tight blood glucose control was widely adopted after the single-center study results of Van den Berghe and colleagues [3], but multicenter studies later suggested that perhaps it was not such an easy approach to apply [4-6] and highlighted the difficulty of translating single-study results to the wider ICU population. But will the pendulum swing back again as automated monitoring systems are developed for continuous and accurate monitoring that will help to reduce the hypoglycemic episodes and as a greater emphasis is placed on avoiding glucose variability rather than on restricting blood glucose to normal levels? 
- aPC attracted much interest with the initial PROWESS (Protein C Worldwide Evaluation in Severe Sepsis) results showing improved outcomes [21]; however, subsequent trial data and concerns about bleeding have dampened initial enthusiasm. These findings led some investigators to challenge the results, and the EMEA requested a second placebo-controlled phase III study [21]. What will the results of the 'repeat' randomized control trial (PROWESS-SHOCK) do to the aPC pendulum?

- Initial excitement regarding the relatively simple approach of aggressive resuscitation using central venous oxygen saturation $\left(\mathrm{ScvO}_{2}\right)$ as a target in a single center [22] has given way to questions about the need for blood transfusions in the resuscitation of patients with sepsis and the overall efficacy of early goaldirected therapy. At present, three large multicenter trials are addressing this question. Will their results also swing the pendulum?

- The use of PEEP has swung from relatively high levels to relatively low levels and back to somewhat higher levels. Will the evidence for low tidal volumes and higher PEEP converge to make high-frequency ventilation an optimal approach to limit VILI?

\section{The next 30 years?}

Given the complex nature of intensive care patients and the disease processes underlying their admission, it seems unlikely that the next 30 years will see the discovery of single therapeutic interventions that, acting alone, will have a major impact on all patients of a given broadly defined class. This is perhaps most apparent for the treatment of patients with sepsis. Mono-therapies for sepsis may be doomed to failure given the multiple redundant and reciprocating autonomic and cellular processes, intracellular pathways, different expression of common injury, variable times of presentation and differing initial clinical status, and variable levels of organ-system reserve, genetic predisposition, and nutritional state. Rather, we will continue to make incremental stepwise advances as our understanding of critical illness continues to expand. Various factors will help in this process. We envision the following:

- Improved communication between basic scientists and ICU physicians will enhance translational research and lead to the development of preclinical models that are more clinically relevant.

- The use of nonlinear complexity models of health and disease will better define disease state and aid development of nonintuitive treatments based on complex organ-system interaction patterns and their resolution in response to therapy. These should provide powerful insights into the basic biology of disease and how our treatments impact on multiple systems.
- There will be a better understanding of the metabolic nature of acute illness as well as metabolic adaptation from subcellular to organ-system levels.

- There will be better identification of patient populations based on genetic factors and biomarkers. Revising our definitions of the phenotypes, such as sepsis and ARDS, with biological and genetic markers may facilitate therapy that is more effective, similar to the way in which some cancers are better managed by appreciation of the clinical phenotype in concert with biological and pathological markers.

- Greater awareness of the time course of the evolving pathophysiology of the underlying disease process and improved diagnostics and genetic profiles of vulnerability will lead to better selection of treatment type and intensity, improved timing of administration and discontinuation, and more sharply targeted therapies. Therapeutic targets will be better defined, based on abnormal, rather than normal, physiology and increased knowledge regarding the limits of adaptation to life-threatening illness. Monitoring relevant physiological variables at the cellular level to detect tolerance or functional distress of the tissues as well as monitoring the response to treatment will facilitate selection of suitable therapies.

- There will be better models to test the effect of complex interventions, often starting from prior to ICU admission and finishing after ICU discharge.

- Better use of functional hemodynamic monitoring principals will guide resuscitation on macrocirculatory and microcirculatory levels.

- There will be less focus on individual aspects of care and a greater emphasis on how different components of the 'package' of ICU treatments work together to improve outcomes.

- There will be better identification of the impact of how health care systems are managed and how care is provided to patient populations and to individuals on the prevalence rates and outcomes of many critical illnesses.

- Improved utilization of electronic tools and technologies will streamline the processes of care delivery. Interactive patient-specific guidelines available at the bedside will assist in decision-making for hemodynamic and respiratory management. Regulatory agencies in various countries will expect clinician compliance with performance metrics based on these guidelines for management of critical illness. There will also be increasing emphasis on reducing demands for blood flow, ventilation, and oxygenation rather than applying potentially noxious therapies to boost their supply.

- Safe and effective mechanical assist devices (for example, left ventricular assist devices and impellers) 
and artificial organ systems (lung, kidney, and liver) will continue to be developed. Incorporation of improved extracorporeal and intravenacaval respiratory gas exchangers into bedside practice will further reduce VILI and minimize or obviate the need for intubation.

- Leveraging of communication technology will extend scarce critical care expertise to underserved settings and improve care uniformity throughout the 24-hour cycle. This may incorporate the use of remote medicine/telemedicine.

- Further focus on perfecting sedation and analgesia stratagems will maintain comfort and near alertness while allowing quality sleep and avoidance of delirium. There will be earlier mobilization to reduce muscle wasting and contractures and to facilitate recovery, and there will be greater input into the management of the long-term sequelae of critical illness.

- A smoother continuum between prehospital care, emergency care, and pre-ICU and post-ICU care, with more interventions beyond the ICU walls, will prevent or accelerate ICU admission and limit complications and 'rebound' following ICU discharge.

- There will be continuing and expanding international collaborations, with the creation of large databases of patients and conduct of multicenter observational and interventional studies.

- Increased efforts will be made to make the ICU more attractive to young physicians and researchers to ensure continued recruitment of enthusiastic and skilled intensivists. Simulation will play an increased role in education and in the development of new skills.

- Basic ICU facilities, training programs, and internetbased decision support tools will be established to improve critical care in developing countries.

We must, however, recognize that these advances in technology and understanding will be challenged by increasing strictures in health-care funding. Intensive care is expensive care. It is thus incumbent upon us not to allow care to be rationed by external forces but to recognize the limitations of what we can offer and when ongoing care is futile. In these cases, we should not needlessly waste resources on prolonging death but should shift the emphasis toward easing the dying process and supporting the patient's family and friends.

\section{Conclusions}

It is difficult to document and quantify the improvements that have been made in the last 30 years. For many problems, mortality rates have not changed much overall; in certain disease processes (for example, sepsis and ARDS), they may have decreased somewhat. However, the population that we are treating in our ICUs has changed and is getting older and sicker. For example, the mean age of ICU patients was over 60 years in recent studies [23,24], so it is difficult to compare current statistics with those of 30 years ago. Given the growing fragility of our patients, even maintaining historical morbidity and mortality rates could signal improvements in care. The aging of populations in many countries will place increasing demands on ICU resources that are already limited and expensive in many areas of the world.

There are clearly areas of intensive care medicine in which we have made little progress and others in which much progress has been achieved. As we look forward to the next three decades of intensive care, it is important to learn from past failures and to build on our successes to create a more effective, efficient, and evidence-based discipline for the future.

\section{Abbreviations}

AKI, acute kidney injury; ALI, acute lung injury; ANZICS, Australian and New Zealand Intensive Care Society; aPC, activated protein C; ARDS, acute respiratory distress syndrome; $\mathrm{CWVH}$, continuous veno-venous hemofiltration; EMEA, European Medicines Agency; ICU, intensive care unit; PEEP, positive end-expiratory pressure; PROWESS, Protein C Worldwide Evaluation in Severe Sepsis; VAP, ventilator-associated pneumonia; VILI, ventilator-induced lung injury.

\section{Competing interests}

The authors declare that they have no competing interests.

\section{Author details}

'Department of Intensive Care, Erasme Hospital, Université libre de Bruxelles, Route de Lennik 808, 1070 Bruxelles, Belgium. ²Department of Intensive Care, University College London, Cruciform Building, Gower Street, London, WC1E 6BT, UK. 3Pulmonary and Critical Care Medicine, Regions Hospital, University of Minnesota, Minneapolis/St. Paul, 640 Jackson Street, St. Paul, MN 55101, USA. ${ }^{4}$ Department of Intensive Care, Centro Hospitalar de Lisboa Central, E.P.E., Alameda de Santo António dos Capuchos, 1169-050 Lisbon, Portugal. 5ivision of Pulmonary, Sleep and Critical Care Medicine, Rhode Island Hospital/ Brown University, 593 Eddy Street, Providence, RI 02903, USA. ${ }^{6}$ Cardiovascular Research Institute and Departments of Medicine and Anesthesia, University of California at San Francisco, 505 Parnassus Ave., San Francisco, CA 94143-0624, USA. ${ }^{7}$ Department of Critical Care Medicine, 606 Scaife Hall, 3550 Terrace Street, Pittsburgh, PA 15261, USA. ${ }^{8}$ Department of Intensive Care Medicine, St George's Healthcare NHS Trust, Blackshaw Road, London, SW17 OQT, UK. Interdepartmental Division of Critical Care Medicine, and Department of Medicine, Division of Respirology, University Health Network and Mt Sinai Hospital, University of Toronto, 600 University Avenue, Suite 18-206, Toronto, ON, M5G 1X5, Canada. ${ }^{10}$ Unit of Critical Care, Faculty of Medicine, Imperial College, London, UK, and Adult Intensive Care Unit, Royal Brompton Hospital, Sydney Street, London, SW6 NP, UK. " Service de Réanimation Polyvalente de I'hôpital Raymond Poincaré, 104 bd Raymond Poincaré, 92380 Garches, France.

${ }^{12}$ Pulmonary and Critical Care Medicine, University of Chicago Hospitals, 5841 S. Maryland Avenue, MC 6026, Chicago, IL 60637, USA.

Published: 27 May 2010

\section{References}

1. Opal SM, Patrozou E: Translational research in the development of novel sepsis therapeutics: logical deductive reasoning or mission impossible? Crit Care Med 2009, 37:S10-S15.

2. Ospina-Tascon GA, Buchele GL, Vincent JL: Multicenter, randomized, controlled trials evaluating mortality in intensive care: doomed to fail? Crit Care Med 2008, 36:1311-1322.

3. Van den Berghe G, Wouters P, Weekers F, Verwaest C, Bruyninckx F, Schetz M, Vlasselaers D, Ferdinande P, Lauwers P, Bouillon R: Intensive insulin therapy in the critically ill patients. N Eng/ J Med 2001, 345:1359-1367.

4. Brunkhorst FM, Engel C, Bloos F, Meier-Hellmann A, Ragaller M, Weiler N, Moerer O, Gruendling M, Oppert M, Grond S, Olthoff D, Jaschinski U, John S, 
Rossaint R, Welte T, Schaefer M, Kern P, Kuhnt E, Kiehntopf M, Hartog C, Natanson C, Loeffler M, Reinhart K: Intensive insulin therapy and pentastarch resuscitation in severe sepsis. N Engl J Med 2008, 358:125-139.

5. Preiser JC, Devos P, Ruiz-Santana S, Melot C, Annane D, Groeneveld J, lapichino G, Leverve X, Nitenberg G, Singer P, Wernerman J, Joannidis M, Stecher A, Chiolero R: A prospective randomised multi-centre controlled trial on tight glucose control by intensive insulin therapy in adult intensive care units: the Glucontrol study. Intensive Care Med 2009, 35:1738-1748.

6. Finfer S, Chittock DR, Su SY, Blair D, Foster D, Dhingra V, Bellomo R, Cook D, Dodek P, Henderson WR, Hebert PC, Heritier S, Heyland DK, McArthur C McDonald E, Mitchell I, Myburgh JA, Norton R, Potter J, Robinson BG, Ronco $\mathrm{JJ}$ : Intensive versus conventional glucose control in critically ill patients. N Engl J Med 2009, 360:1283-1297.

7. The Acute Respiratory Distress Syndrome Network: Ventilation with lower tidal volumes as compared with traditional tidal volumes for acute lung injury and the acute respiratory distress syndrome. N Eng/ J Med 2000, 342:1301-1308.

8. Erickson SE, Martin GS, Davis JL, Matthay MA, Eisner MD: Recent trends in acute lung injury mortality: 1996-2005. Crit Care Med 2009, 37:1574-1579.

9. Bellomo R, Cass A, Cole L, Finfer S, Gallagher M, Lo S, McArthur C, McGuinness S, Myburgh J, Norton R, Scheinkestel C, Su S: Intensity of continuous renalreplacement therapy in critically ill patients. N Eng/ J Med 2009, 361:1627-1638.

10. Hebert PC, Wells G, Blajchman MA, Marshall J, Martin C, Pagliarello G, Tweeddale M, Schweitzer I, Yetisir E: A multicenter, randomized, controlled clinical trial of transfusion requirements in critical care. Transfusion Requirements in Critical Care Investigators, Canadian Critical Care Trials Group. N Engl J Med 1999, 340:409-417.

11. Strom T, Martinussen T, Toft P: A protocol of no sedation for critically ill patients receiving mechanical ventilation: a randomised trial. Lancet 2010, 375:475-480.

12. Vincent JL: Give your patient a fast hug (at least) once a day. Crit Care Med 2005, 33:1225-1229.

13. Amerling R, Winchester JF, Ronco C: Guidelines have done more harm than good. Blood Purif 2008, 26:73-76.

14. Wiedemann HP, Wheeler AP, Bernard GR, Thompson BT, Hayden D, deBoisblanc B, Connors AF Jr., Hite RD, Harabin AL: Comparison of two fluidmanagement strategies in acute lung injury. N Engl J Med 2006, 354:2564-2575.
15. Finfer S, Bellomo R, Boyce N, French J, Myburgh J, Norton R: A comparison of albumin and saline for fluid resuscitation in the intensive care unit. N Engl J Med 2004, 350:2247-2256.

16. Harvey S, Harrison DA, Singer M, Ashcroft J, Jones CM, Elbourne D, Brampton W, Williams D, Young D, Rowan K: Assessment of the clinical effectiveness of pulmonary artery catheters in management of patients in intensive care (PAC-Man): a randomised controlled trial. Lancet 2005, 366:472-477.

17. De Backer D, Biston P, Devriendt J, Madl C, Chochrad D, Aldecoa C, Brasseur A, Defrance P, Gottignies P, Vincent JL: Comparison of dopamine and norepinephrine in the treatment of shock. N Engl J Med 2010, 362:779-789.

18. Dellinger RP, Levy MM, Carlet JM, Bion J, Parker MM, Jaeschke R, Reinhart K, Angus DC, Brun-Buisson C, Beale R, Calandra T, Dhainaut JF, Gerlach H, Harvey M, Marini JJ, Marshall J, Ranieri M, Ramsay G, Sevransky J, Thompson BT, Townsend S, Vender JS, Zimmerman JL, Vincent JL: Surviving Sepsis Campaign: international guidelines for management of severe sepsis and septic shock: 2008. Crit Care Med 2008, 36:296-327.

19. Vincent JL: Steroids in sepsis: another swing of the pendulum in our clinical trials. Crit Care 2008, 12:141.

20. Sprung CL, Annane D, Keh D, Moreno R, Singer M, Freivogel K, Weiss YG, Benbenishty J, Kalenka A, Forst H, Laterre PF, Reinhart K, Cuthbertson BH, Payen D, Briegel J: Hydrocortisone therapy for patients with septic shock. NEngl J Med 2008, 358:111-124.

21. Bernard GR, Vincent JL, Laterre PF, LaRosa SP, Dhainaut JF, Lopez-Rodriguez A, Steingrub JS, Garber GE, Helterbrand JD, Ely EW, Fisher CJ Jr.: Efficacy and safety of recombinant human activated protein $\mathrm{C}$ for severe sepsis. NEngl J Med 2001, 344:699-709.

22. Rivers E, Nguyen B, Havstad S, Ressler J, Muzzin A, Knoblich B, Peterson E, Tomlanovich M: Early goal-directed therapy in the treatment of severe sepsis and septic shock. N Engl J Med 2001, 345:1368-1377.

23. Vincent JL, Sakr Y, Sprung CL, Ranieri VM, Reinhart K, Gerlach H, Moreno R, Carlet J, Le Gall JR, Payen D: Sepsis in European intensive care units: results of the SOAP study. Crit Care Med 2006, 34:344-353.

24. Vincent JL, Rello J, Marshall J, Silva E, Anzueto A, Martin CD, Moreno R, Lipman J, Gomersall C, Sakr Y, Reinhart K: International study of the prevalence and outcomes of infection in intensive care units. JAMA 2009, 302:2323-2329.

doi:10.1186/cc8979

Cite this article as: Vincent J-L, et al:: Thirty years of critical care medicine. Critical Care 2010, 14:311. 